\title{
Invited Commentary on "Probiotics in Paediatric Practice" by Vuletic et al.: Let us Use Scientific Evidence for the Benefit of Our Patients
}

\author{
Elena Daniela Serban \\ $2^{\text {nd }}$ Department of Pediatrics, "Iuliu Hatieganu" \\ University of Medicine \& Pharmacy, Emergency \\ Clinical Hospital for Children, Cluj-Napoca, \\ Romania \\ Correspondence: \\ daniela.serban@umfcluj.ro \\ Tel.: + (40) 264-532216 \\ Fax.: + (40) 264-590478
}

Received: January 27, 2019

Accepted: January 30, 2018

Key Words: Probiotics - Scientific Evidence - Efficacy - Safety.

Probiotics represent one of the hottest topics in medicine over the past 10 years, many scientific manuscripts being published on a daily basis, with more than 23400 papers on PubMed. Consequently, in Europe, as across the whole globe, probiotics' sales and consumption have increased tremendously. There has been an exponential growth in the number of commercial products, about a hundred probiotic products being available on the market in many European countries. Therefore, it is difficult for doctors to remain up to date and to choose the best probiotic and when to use it. The most important misconceptions I encounter in my practice are that "all probiotics are considered equal" and "the more strains in a product, the bet- ter". We live in an era where the probiotic industry is quite assertive, through the mass media, websites, and brochures distributed to doctors and consumers. Consequently, many probiotics are categorised as "the best", "top number 1", "the most innovative", "the best scientifically documented bacterial strain in the world", etc. First, this industry promotes the "probiotic umbrella" concept. That means to take advantage of results obtained from a specific probiotic by extending them to other products, blurring the specificity of those products, dose, duration of intake, combination of strains, and methods used to manufacture the formulation with which the benefits were obtained (1). Due to extrapolations of indications, all probiotics are recommended in all conditions, often without any scientific evidence of efficacy, just because they are "trendy" or they are "healthy bacteria". Second, the probiotic industry usually presents studies showing the efficacy and/or safety of one or two strains in a multistrain product, with no study being performed on the whole mixture. Companies try to convince us that products containing several strains, with applicability in various conditions, are universally useful. However, the contrary may be true: together, these strains may counteract each other's positive effects. 
The review by Vuletic et al. (2) is wonderful in clarifying these misconceptions, shedding lights on doctors' dilemmas. The authors present clearly the positive, controversial and nonindications for probiotic use (prevention and/ or therapy) in most common paediatric conditions. The recommendations are based on high-quality scientific evidence, including The European Society for Paediatric Gastroenterology, Hepatology and Nutrition (ESPGHAN) Working Group (WG) for Probiotics and Prebiotics and the European Crohn's and Colitis Organization (ECCO) and ESPGHAN guidelines. When there are no guidelines available, the authors present the results of the randomized controlled trials (RCTs) available for each topic. Despite the existence of so many products, the authors demonstrate that efficacy and safety are strain and dose specific, and only a few specific strains (Lactobacillus GG - LGG, Lactobacillus reuteri DSM 17938, Saccharomyces boulardii and Escherichia coli Nissle 1917) have been proven effective and safe in preventing and/or treating specific conditions. Paediatricians should also notice that probiotics have no proven efficacy in functional constipation and Crohn's disease, in spite of their frequent use in practice. The review by Vuletic et al. (2) included another excellent paper regarding guidance on the use of probiotics in clinical practice in children (3), but added more specific clinical conditions (such as Helicobacter pylori infection, inflammatory bowel disease and necrotizing enterocolitis in preterm infants) and more recent RCTs.

We definitely have good scientific evidence. However, experience with probiotics is evolving and we have to be aware of the new data. Very recently, Freedman et al. (4) (Canada, 886 children) and Schnadower et al. (5) (USA, 971 children) compared the effectiveness of probiotics in two multicentre prospective, randomized, double-blind, placebo-controlled trials, in children (3-48 months of age) with acute gastroenteritis. Probiotics [combined Lactobacillus rhamno- sus R0011 and Lactobacillus helveticus R0052 - $4 \times 10^{9}$ colony-forming units twice daily (4), respectively LGG - 1 x $10^{10}$ colony-forming units twice daily (5)] were administered for 5 days. Both high-quality trials showed that probiotics were not significantly better than the placebo for all outcomes. The results are disappointing; however, we need more data from a European experience.

Until then, the review by Vuletic et al. (2) represents a very useful and comprehensive review for our paediatric practice. Paediatricians' awareness / conscience should stand against the aggressiveness of the probiotic industry and always use only the strains that have been proven effective and safe in welldesigned RCTs, for the benefit of their patients, with no harm. Probiotics which are "really friendly, not just trendy" should be used. In choosing the right strain at the right dose, there is no "one size fits all".

Disclosure: Dr. Serban has participated as a clinical investigator and/or advisory board member and/or consultant and/or speaker for Abbvie, Montavit, Nestle, Noventure, Nutricia and Reckitt Benckiser.

\section{References}

1. de Simone C. The Unregulated Probiotic Market. Clin Gastroenterol Hepatol. Epub 14/03/2018. doi: 10.1016/j.cgh.2018.01.018. PubMed PMID: 29378309.

2. Vuletic B, Kocovic A, Mladenovic M, Radlovic V, Djindjic J, Radlovic J. Probiotics in Paediatric Practice. Central Eur J Paed. 2019;15(1):1-14.

3. Hojsak I, Fabiano V, Pop TL, Goulet O, Zuccotti $\mathrm{GV}$, Çokuğraş FC, et al. Guidance on the use of probiotics in clinical practice in children with selected clinical conditions and in specific vulnerable groups. Acta Paediatr. 2018;107(6):927-37.

4. Freedman SB, Williamson-Urquhart S, Farion KJ, Gouin S, Willan AR, Poonai N, et al. Multicenter trial of a combination probiotic for children with gastroenteritis. N Engl J Med. 2018;379:2015-26.

5. Schnadower D, Tarr PI, Casper TC, Gorelick MH, Dean JM, O'Connell KJ, et al. Lactobacillus rhamnosus GG versus placebo for acute gastroenteritis in children. N Engl J Med. 2018;379:2002-14. 